\title{
Acute appendicitis revisited: computed tomography and ultrasound scan are they necessary to establish a diagnosis?
}

\author{
Osman A Hamour ${ }^{*}$, Eman M.Fallatah ${ }^{1}$, Rawan O. Alshehri ${ }^{1}$, Zain A. Alshareef ${ }^{1}$ and Halah F.AL-Enizi ${ }^{2}$ \\ ${ }^{1}$ King Faisal Specialist Hospital, Jeddah, Saudi Arabia \\ ${ }^{2}$ Research Center, Jeddah, Saudi Arabia
}

\begin{abstract}
Background: Acute appendicitis is a common and challenging disease. It represents one of the most common emergency surgical diseases encountered worldwide. It affects more than 250,000 people annually. It is globally rated as number one acute surgical emergency. The gold-standard for the diagnosis of acute appendicitis remains to be a properly taken history and a thoroughly conducted physical examination. However, recently Computed Tomography (CT) and Ultrasound Scan (USS) have been described as accurate diagnostic modalities in patients suspected to have acute appendicitis. Yet the role of CT scan in patients suspected to have acute appendicitis, but with equivocal clinical picture remains ill defined.

Methods: This is a retrospective study conducted in King Faisal Specialist Hospital and Research Center, Jeddah, Saudi Arabia. The medical charts of patients who underwent an appendicectomy between first January 2010 and the end of February 2015 were reviewed for demographic data, clinical diagnosis, laboratory tests, imaging, and the type of surgery performed, the complications, and the ultimate outcome. The collected data was entered in a Case Review Form (CRF). The patients were divided in two groups (1 and 2 ) according to the final histopathology result. Group 1 had a normal appendix and group 2 had acute appendicitis. The two groups were compared according to the above mentioned parameters.

Results: Ninety patients were enrolled, 48 (53.3\%) were males. Sixty six (73.3\%) patients underwent laparoscopic appendicectomy and 24 (26.7\%) patients underwent open appendicectomy. The histopathological diagnosis of acute appendicitis was made in 78 (86\%) patients, and 12 (13\%) patients had a normal appendix. The accuracy of the clinical diagnosis of acute appendicitis was $80 \%$, while that of the CT scan and USS were 78\% and $44 \%$, respectively.

Conclusion: In our study and other cited studies, a good clinical examination remains the gold standard for the diagnosis of acute appendicitis. It carries the highest sensitivity and the highest accuracy rate. USS, however, continues to be an important tool in the evaluation of acute gynecologic conditions, while CT scan is thought to be rapidly and confidently making an accurate diagnosis in equivocal cases.
\end{abstract}

\section{Introduction}

Acute appendicitis is well recognized as the most common surgical cause of acute abdomen with a life time risk for developing acute appendicitis to be $8.6 \%$ for men and $6.7 \%$ for women [1]. The goldstandard for a correct diagnosis of acute appendicitis remains to be a good history and a thoroughly conducted physical examination. This well-established clinical diagnosis though labeled as the gold-standard, it is still attended by what surgeons call an acceptable negative appendecectomy rate of $7 \%$ to $25 \%$ [2,3]. Having said this one needs to know that the classical clinical presentation of acute appendicitis exemplified in generalized or periumblical pain that migrates to settle in the right iliac fossa associated with anorexia, nausea, vomiting, tenderness with rebound tenderness in the right iliac fossa and mild elevation of temperature with leukocytosis, may only be found in $20 \%$ of patients. There is a wide range of differential diagnosis when acute appendicitis presents in an atypical manner. In such circumstances more investigations may be required to rule out other causes of acute abdomen. The management of such atypical cases has been the focus of much debate; therefore when surgical intervention is performed in this group of patients the negative appendecectomy rate approaches $50 \%$ [4]. In such circumstances adjunctive diagnostic tools might be utilized to help reaching a correct diagnosis. USS has been utilized as a diagnostic modality in this subgroup of patients, with the criticism that it is operator dependent, and that its use is primarily limited to rule out gynecological pathology. On the other hand a high resolution CT has been reported to be accurate in patients with acute appendicitis $[5,6]$. In most of the general hospitals missing a diagnosis of acute appendicitis is the most common medico-legal issue. Such a delay in the diagnosis of acute appendicitis increases the risk of perforation which, consequently increases the risk of postoperative complications to $39 \%$ as compared to $8 \%$ for simple appendicitis [7]. This is why these diagnostic imaging tools have proved to be quite helpful in equivocal cases and for the elucidation of other causes of acute abdominal pain [8].

The CT scan and USS are the current imaging modalities of choice that can provide a rapid and effective evaluation of lower abdominal pain in equivocal cases. The CT scan gives a diagnosis of acute appendicitis by showing a distended appendix, an appendicolith, infiltration of peri-appendiceal fat, focal thickening of the base of the caecum and an appendix diameter of greater than $6 \mathrm{~mm}$. The USS in addition to it being useful to rule out pelvic pathology, and being mostly operator dependent it can also demonstrate the presence of

Correspondence to: Osman Hamour, King Faisal Specialist Hospital, Jeddah, Saudi Arabia, E-mail: osmanahamour@gmail.com, ohamour@kfshrc.edu.sa

Key words: acute appendicitis, ad hoc computerized tomography, ultrasound scan, clinical diagnosis

Received: June 04, 2016; Accepted: June 20, 2016; Published: June 23, 2016 
probe tenderness and rebound tenderness an extra sign in favor of acute appendicitis [9].

\section{Methods}

This is a retrospective study conducted in King Faisal Specialist Hospital and Research Center, a tertiary care institution in Jeddah, Kingdom of Saudi Arabia. After the Institutional Review Board (IRB) approval was granted the charts of patients who underwent an appendecectomy between the first of January 2010 and the end of February 2015 were reviewed and entered in a case review form (CRF). Patients who presented with symptoms and signs suggestive of acute appendicitis are initially assessed and investigated by the emergency room physician (ER) The ER physician takes the patient history, conducts a clinical examination, documents all the findings, writes a provisional diagnosis, requests relevant laboratory tests and images according to his/her own impression and discretion because there is no clinical pathway or a guideline protocol for the ER physician to follow, regarding when to order a CT scan or an USS, nor when to refer the patient to the surgical team. The ER physician then according to his/ her judgment refers to the surgical team all the patients that he/she believes that they have a definite acute appendicitis or even those who have atypical appendicitis and those patients who have appendicitis in the differential diagnosis and who deserve a surgical consultation. Once consulted, the surgical team will take over the patient for further management. This includes, preoperative care, requesting more labs and images, surgical intervention, postoperative care, and antibiotics and fluid therapy. The question whether the patient has typical or atypical acute appendicitis is always resolved by the ER physician in charge even before the surgical team is informed by ordering an ad hoc USS if the patient is a female or a CT scan if the patient is a male and some cases both tests are requested, again following no specific protocol. All the patients who had an appendecectomy for a diagnosis of acute appendicitis were included in the study. The patients who satisfied the inclusion criteria were divided in two groups according to the final histopathology diagnosis. Group 1 had a normal appendix and group 2 had an inflamed appendix. The two groups were compared as regard to the clinical diagnosis, the laboratory results, the imaging performed, the final diagnosis and whether it was supported by imaging, the type of surgery performed the outcome of treatment, the disposition, the hospital stay, and any complications reported. Patients with another diagnosis or a differential diagnosis of pain in the right iliac fossa not due to acute appendicitis were excluded as well as patients with incomplete or missing charts. Significance for all analyses was set at a $P$ value $<0.05$. All data analysis was performed using SPSS version 20.

\section{Results}

Ninety patients had an appendicectomy performed between the first of January 2010 and the end of February 2015 at the King Faisal Special Hospital and Research Center, Jeddah, Kingdom of Saudi Arabia. Forty eight (53\%) patients were males with a mean age $28 \pm$ 16 years.

Sixty six (73\%) patients had laparoscopic appendicectomy, and 24 (27\%) patients had open appendicectomy. Eighty one (90\%) patients were discharged home on the second postoperative day. Regarding presenting symptoms, sixty seven $(74.4 \%)$ patients had a history of periumblical or generalized abdominal pain shifted to the right iliac fossa. Twenty eight (31.1\%) patients had low grade fever, 18 (20\%) had anorexia, $50(55.6 \%)$ had nausea, $41(45.6 \%)$ had vomiting, 10 (11.1\%) had diarrhea, and 3 (3.2\%) patients had constipation. Physical examination showed that $22(24.4 \%)$ patients had tenderness in the right lower quadrant, and 61(67.8\%) had rebound tenderness. Histopathological examination showed that seventy eight (87\%) patients had acute appendicitis on histopathological examination and $12(13 \%)$ patients had a normal appendix.

Figure 1 shows the total number of patients who had positive or negative clinical signs of acute appendicitis by using an USS and/or CT scan to augment the diagnosis.

Out of the 90 patients, 80 had either CT scan alone or USS alone or both, 28 (31\%) patients had CT scan only, 31 (34\%) had USS only, $21(23 \%)$ had both CT scan and USS, and $10(11 \%)$ patients diagnosed clinically only. Fifty two (57\%) patients underwent preoperative USS, it showed a picture of acute appendicitis in $22(42 \%)$ patients, while $30(58 \%)$ patients were negative for acute appendicitis. In contrast, CT scanscan, which was performed in 49 (54\%) patients, showed a picture of acute appendicitis in $37(76 \%)$ and the rest $12(24 \%)$ were negative for acute appendicitis.

According to histopathology, patients with normal appendix (Group 1) were compared with patients with inflamed appendix (Group 2) regarding the clinical and the histological diagnosis, the laboratory results, the imaging studies performed, the type of surgery done, and the complications. Both clinical findings and WBC $>10,000$

Table 1. Comparison of patients with normal appendix (group 1) and patients with inflamed appendix (group 2) according to histopathology regarding clinical diagnosis, labs done, imaging diagnosis, type of surgery and complication.

\begin{tabular}{|c|c|c|c|}
\hline \multirow[t]{2}{*}{ Parameters } & \multicolumn{2}{|c|}{ Groups according to histopathology } & \multirow[t]{2}{*}{ P-value } \\
\hline & $\begin{array}{l}\text { Group } 1(\mathrm{~N}=12) \\
\text { Patients with normal appendix }\end{array}$ & $\begin{array}{c}\text { Group } 2(\mathrm{~N}=78) \\
\text { Patients with inflamed appendix }\end{array}$ & \\
\hline Clinical findings of acute appendicitis & $7(58 \%)$ & $67(85 \%)$ & 0.02 \\
\hline $\begin{array}{l}\text { Blood tests } \\
\qquad \begin{array}{l}\text { WBC }>10,000 \\
\\
\text { Neutrophilia }(>75 \%)\end{array}\end{array}$ & $\begin{array}{l}4(33 \%) \\
3(25 \%)\end{array}$ & $\begin{array}{l}60(76 \%) \\
45(57 \%)\end{array}$ & $\begin{array}{l}0.004 \\
0.059\end{array}$ \\
\hline $\begin{array}{l}\text { Imaging showed picture of appendicitis } \\
\text { CT } \\
\text { US } \\
\text { Both }\end{array}$ & $\begin{array}{c}4(33 \%) \\
3(25 \%) \\
1(8 \%)\end{array}$ & $\begin{array}{l}33(42 \%) \\
19(24 \%) \\
2(2.5 \%)\end{array}$ & $\begin{array}{c}0.75 \\
1 \\
0.35\end{array}$ \\
\hline $\begin{array}{l}\text { Types of surgery } \\
\text { Lap } \\
\text { Open }\end{array}$ & $\begin{array}{l}8(66 \%) \\
4(33 \%)\end{array}$ & $\begin{array}{l}58(74 \%) \\
20(25 \%)\end{array}$ & $\begin{array}{l}0.73 \\
0.73\end{array}$ \\
\hline Complications & None & None & ---- \\
\hline
\end{tabular}


showed statistical significant difference, $p$-values $=0.02$ and 0.004 , respectively (Table 1).

The CT scan and the USS results were verified by the histopathology diagnosis. Out of the 12 patients in the normal histopathology group, only 4 patients had both CT and USS. One patient had both true positive CT and USS, two patients had both true negative and one patient had false positive USS and true negative CT. On the other hand, out of the 78 patients with a positive histopathology of acute appendicitis, only $17(22 \%)$ patients had both CT and USS. Four patients had the same results in CT scan and USS, two patients had both true positive CT and USS and the other two patients showed both CT and USS false negative results and 13 patients had true positive CT and USS false negative results (Table 2).

The clinical assessment supported by CT scan or not and confirmed by final histopathology as shown in (Table 3). Four (44\%) out of 9 patients in the normal histopathology group had the same result in clinical finding and CT scan ( 2 false positive results and 2 true negative results). In patients with a positive histopathology of acute appendicitis, $33(82 \%)$ out of 40 patients had similar result in clinical finding and CT scan (31 true positive results and 2 false negative results). Furthermore, the clinical assessment supported by USS or not and confirmed by final

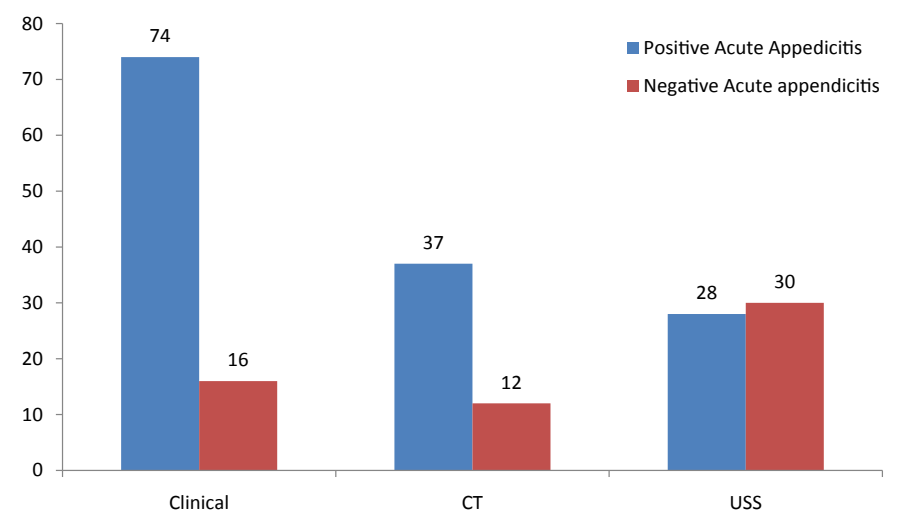

Figure 1. Shows the number of patients who were clinically diagnosed as acute appendicitis supported by CT scan and/or an USS or either of them was not done. histopathology as shown in (Table 4). Four (57\%) out of 7 patients in the normal histopathology group, had similar result in clinical finding and USS ( 3 false positive of acute appendicitis and one true negative result). In patients with a positive histopathology of acute appendicitis, 19 (42\%) out of 45 patient had the same result in clinical finding and USS (16 true positive for acute appendicitis and 3 false negative results). The main outcome measure included; clinical assessment had a sensitivity of $86 \%$, a specificity of $42 \%$, The CT scan on the other hand had a sensitivity of $83 \%$, a specificity of $56 \%$, while the USS had a sensitivity of $42 \%$, a specificity of $57 \%$, (Table 5 ).

\section{Discussion}

Although the treatment of acute appendicitis is simple and straightforward, its diagnosis remains a challenge, and the negative appendicectomy rate in large series ranges from $15 \%$ to $33 \%$ [10]. This figure rises to $45 \%$ in patients with atypical clinical picture and during child bearing age [11]. The purpose of this study is to determine the impact of an ad hoc CT scan and/or an USS as adjunctive diagnostic tools in patients who were admitted with a possible diagnosis of acute appendicitis in our emergency medicine department. The final verification reference diagnosis was obtained by histopathology. The question when to do a CT scan and / or an USS to diagnose acute appendicitis and to verify their ability to rule out other pelvic causes of acute abdomen is significant. The number of cases enrolled in this study is 90 patients only collected over 5 years, this we believe, can be explained by the fact that our hospital is a tertiary care hospital and the cases of appendicitis that present to us are only the employees, their dependents and the patients who are eligible for treatment in the hospital for another disease. Whether this low volume would affect the ability to diagnose these cases or it is a reason for depending on imaging for diagnosis, is yet to be verified by another study designed for such a purpose.

The history of migratory pain, anorexia, nausea, vomiting, right iliac fossa tenderness, rebound tenderness, and leukocytosis, remain reliable and accurate diagnostic findings in patients suspected of having acute appendicitis. When acute appendicitis presents in this classic form, it is easily diagnosed and should be treated without the aid of further imaging modalities. However during the last decade, studies of USS and CT scan have suggested that these diagnostic imaging modalities

Table 2. Describes the two groups of patient divided according to the histopathology results, by using CT scan and/ or USS as an imaging modality or weather was not done.

\begin{tabular}{|c|c|c|c|c|c|c|c|c|c|c|c|c|}
\hline \multirow[t]{4}{*}{ US } & \multicolumn{8}{|c|}{ Histopathology } & \multicolumn{4}{|c|}{ Total (US vs. CT) } \\
\hline & \multicolumn{4}{|c|}{ Normal appendix } & \multicolumn{4}{|c|}{ Acute appendicitis } & & & & \\
\hline & \multicolumn{4}{|c|}{ CE CT } & \multicolumn{4}{|c|}{ CT } & & & & \\
\hline & $+\mathrm{ve}$ & - ve & Not done & Total & $+v e$ & - ve & Not done & Total & $+\mathrm{ve}$ & - ve & Not done & Total \\
\hline Positive & 1 & $\underline{1}$ & 1 & 3 & $\underline{\mathbf{2}}$ & $\underline{\mathbf{0}}$ & 17 & 19 & $\underline{\mathbf{3}}$ & 1 & 18 & 22 \\
\hline Negative & $\underline{\mathbf{0}}$ & $\underline{2}$ & 2 & 4 & $\underline{13}$ & $\underline{2}$ & 11 & 26 & $\underline{13}$ & $\underline{4}$ & 13 & 30 \\
\hline Not done & 3 & 2 & 0 & 5 & $\overline{18}$ & $\overline{5}$ & 10 & 33 & $\overline{21}$ & $\overline{7}$ & 10 & 38 \\
\hline Total & 4 & 5 & 3 & 12 & 33 & 7 & 38 & 78 & 37 & 12 & 41 & 90 \\
\hline
\end{tabular}

Table 3. Describes the two groups of patients who according to histopathology result were clinically diagnosed as acute appendicitis or the diagnosis was negative clinically supported by $\mathrm{CT}$ scan as an imaging modality or the CT scan was not done.

\begin{tabular}{|c|c|c|c|c|c|c|c|c|c|c|c|c|}
\hline \multirow[t]{4}{*}{ Clinical } & \multicolumn{8}{|c|}{ Histopathology } & \multirow{2}{*}{\multicolumn{4}{|c|}{ Total (Clinical vs. CT) }} \\
\hline & \multirow{2}{*}{\multicolumn{4}{|c|}{$\begin{array}{c}\text { Normal appendix } \\
\text { CT }\end{array}$}} & \multicolumn{4}{|c|}{ Acute appendicitis } & & & & \\
\hline & & & & & & & & & \multirow[b]{2}{*}{+ ve } & & \multirow[b]{2}{*}{ Not done } & \multirow[b]{2}{*}{ Total } \\
\hline & $+\mathrm{ve}$ & -ve & Not done & Total & + ve & -ve & Not done & Total & & -ve & & \\
\hline \multirow[t]{2}{*}{ Positive Negative } & $\underline{2}$ & $\underline{\mathbf{3}}$ & 2 & 7 & $\underline{31}$ & $\underline{\mathbf{5}}$ & 31 & 67 & $\underline{33}$ & $\underline{8}$ & 33 & 74 \\
\hline & $\underline{2}$ & $\underline{2}$ & 1 & 5 & $\underline{\mathbf{2}}$ & $\underline{2}$ & 7 & 11 & $\underline{4}$ & $\underline{4}$ & 8 & 16 \\
\hline Total & 4 & 5 & 3 & 12 & 33 & 7 & 38 & 78 & 37 & 12 & 41 & 90 \\
\hline
\end{tabular}


Table 4. Describes the two groups of patients according to histopathology result as normal or acute appendicitis, patients were clinically diagnosed as acute appendicitis or the diagnosis was clinically negative, supported by USS as an imaging modality or the test was not done.

\begin{tabular}{|c|c|c|c|c|c|c|c|c|c|c|c|c|}
\hline \multirow[t]{4}{*}{ Clinical } & \multicolumn{8}{|c|}{ Histopathology } & \multirow{3}{*}{\multicolumn{4}{|c|}{ Total (Clinical vs. US) }} \\
\hline & \multirow{2}{*}{\multicolumn{4}{|c|}{$\begin{array}{c}\text { Normal appendix } \\
\text { US }\end{array}$}} & \multicolumn{4}{|c|}{ Acute appendicitis } & & & & \\
\hline & & & & & \multicolumn{4}{|c|}{ US } & & & & \\
\hline & $+\mathrm{ve}$ & -ve & Not done & Total & $+\mathrm{ve}$ & -ve & Not done & Total & $+\mathrm{ve}$ & -ve & Not done & Total \\
\hline $\begin{array}{l}\text { Positive } \\
\text { Negative }\end{array}$ & $\underline{\mathbf{3}}$ & $\begin{array}{l}\underline{3} \\
\underline{1}\end{array}$ & $\begin{array}{l}1 \\
4\end{array}$ & $\begin{array}{l}7 \\
5\end{array}$ & $\frac{16}{\underline{3}}$ & $\frac{23}{\underline{3}}$ & $\begin{array}{c}28 \\
5\end{array}$ & $\begin{array}{l}67 \\
11\end{array}$ & $\begin{array}{c}19 \\
3\end{array}$ & $\frac{26}{4}$ & $\frac{29}{9}$ & $\begin{array}{l}74 \\
16\end{array}$ \\
\hline Total & 3 & 4 & 5 & 12 & 19 & 26 & 33 & 78 & 22 & 30 & 38 & 90 \\
\hline
\end{tabular}

Table 5. compares the PPV, NPV, Sensitivity, specificity and accuracy among all the utilized diagnostic tools in patients suspected with acute appendicitis.

\begin{tabular}{|l|c|c|c|}
\hline Test & Clinical & CT Scan & USS \\
\hline Sensitivity (95\% CI) & $86(0.78-0.94)$ & $83(0.71-0.94)$ & $56(0.23-0.88)$ \\
\hline Specificity (95\%CI) & $42(0.14-0.70)$ & $78(0.66-0.89)$ & $57(-0.20-0.94)$ \\
\hline Accuracy (95\%CI) & $80(0.72-0.88)$ & $89(079-0.99)$ & $44(0.31-0.58)$ \\
\hline PPV (95\%CI) & $91(0.84-0.97)$ & $42(0.14-0.70)$ & $86(0.72-1.01)$ \\
\hline NPV (95\%CI) & $31(0.09-0.54)$ & $13(0.01-0.25)$ & \\
\hline
\end{tabular}

may improve the diagnostic accuracy of acute appendicitis [12]. On the other hand, there were concerns about the appropriateness and accuracy of these modalities without surgical evaluation [13]. Although USS has an excellent performance in prospective studies with a mean sensitivity $86 \%$ (our study $42 \%$ ) and a median specificity of $96 \%$ (our study $57 \%$ ) in diagnosing appendicitis, it has the limitation of variable reliability and its well-known operator dependency. The latter is quite obviously seen in comparing the USS sensitivity and specificity in our study with the other study quoted above. USS is well known to incur low cost, it lacks ionizing radiation, or need of patient's preparation, and the ability to provide dynamic information through graded compression and moreover it is non-invasive, yet the test adds cost and may delay surgical intervention. Therefore, it appears that USS may be more useful in excluding potential pelvic pathology in equivocal cases, but there are little data available to unequivocally support the benefits of USS in patients with classic clinical symptoms and signs of acute appendicitis. In our study $52(57 \%)$ patients underwent preoperative USS; it showed a picture of acute appendicitis in 22 (24\%) patients, while $30(33 \%)$ patients were negative for acute appendicitis.

The advantages of CT scan include; less operator dependent than USS, enhanced delineation of the extent of the disease in the case of perforated appendicitis, easier visualization of a retro-caecal appendix, unchanged quality of imaging regardless of, the presence of bowel gas, obesity or severe abdominal pain, and the possibility of multipanar retrospective data reconstruction. The CT scan has been described as an accurate diagnostic imaging modality in patients suspected with acute appendicitis and equivocal findings. In clinical trials, CT scan has been superior to USS in terms of diagnostic accuracy and reliability in atypical presentations and can be specific and sensitive in diagnosing classic acute appendicitis [9]. In our study CT scan was more superior in diagnosing acute appendicitis compared to USS. It was performed in $49(54 \%)$ patients, $37(76 \%)$ were positive for acute appendicitis and 12 (24\%) were negative. However, most authors do not support the use of CT scan alone, but recommend that CT scan result be correlated with the clinical picture [14].

In our study, the positive predictive value for clinical findings consistent with acute appendicitis was $91 \%$, a rate that can be achieved with taking a good history and thorough physical examination, the majority of surgeons agree that the next step in managing the patients with positive clinical findings of acute appendicitis is to take the patient for appendicectomy. Since further imaging studies in such patients do not help and they may significantly prolong the emergency department evaluation time and consequently delay a badly needed appendicectomy. If not timely done unwarranted complications like appendiceal rupture will ensue. Such complications can be minimized by prompt and accurate diagnosis. The incidence of appendiceal perforations ranges from 17 to $40 \%$ [15]. A higher rate of rupture up to $70 \%$ is seen in elderly patients, children, patients with delayed medical care, and women of the child bearing age [16]. The mortality rate from a ruptured appendix ranges from 0.17 to $7.5 \%$ for adults and can be as high as $20 \%$ for children under the age of 2 years [17]. In our series we did not have children neither we had any mortality or morbidity.

On the other hand patients with atypical presentation should be hospitalized for active observation including frequent clinical assessment and further imaging studies until a diagnosis is reached, the patient discharged or else a diagnostic laparoscopy is done. Given its excellent diagnostic yield, zero mortality, low morbidity, and easy availability, one should consider the early use of diagnostic laparoscopy instead of CT scan, USS or observation in patients suspected of having acute appendicitis but with atypical presentation. In our study, out of 90 patients with suspected acute appendicitis 66 patients had a laparoscopic appendecectomy, 8 of them were normal appendices and 58 were inflamed. In none of the patients' chart was it written whether the laparoscopy was meant to be diagnostic or therapeutic or both, although the end result is the same, as most patients who go for diagnostic laparoscopy, the appendix will be removed any way. However, for a scientific study it can make a difference especially that the accuracy is on the variables of the study. Taking the general wisdom and common sense that the diagnosis of atypical appendicitis should be a common and every day practice for any surgeon who has seen cases with lower abdominal pain, yet some people might argue that specific criteria must be cited, in order to define what is meant by atypical appendicitis, what investigations, images, and observational protocol to be followed and when to interfere surgically or when to discharge the patient. Our study being retrospective citing criteria for defining what an atypical appendicitis is was not possible. Such criteria should instate when to order an USS, a CT scan, do a diagnostic laparoscopy, or open surgery. One of the limitations of our retrospective study is that the decision to obtain an USS and / or a CT scan is entirely under the control of the ER physician and it follows no specific pathway or protocol, the ER physician's intention may not be limited to diagnosing appendicitis as he may also wanted to rule out other diseases, or else the imaging may 
have been ordered to confirm typical acute appendicitis presentation. In fact, many patients, even with classical clinical indications of the presence or absence of appendicitis, still received a CT scan evaluation. Twelve patients in our study had a histologically normal appendix, 7 of them had a clinical diagnosis of acute appendicitis, and CT scan showed an acute appendicitis in 4 patients and USS in 3 patients and 1 patient had a positive CT scan and a positive USS though the appendix was histologically normal. This is probably another limitation in the study of having no control on the images' ordering. Here image studies were done in spite of a positive clinical examination. The lack of control over the decision to order a CT scan creates the difficulty of categorically identifying which CT scan was ordered only to evaluate appendicitis. Another limitation to the study is the question of proper documentation and accuracy of the history taken and the interpretation of physical signs. Unlike in other studies when the classical clinical picture of acute appendicitis, described above, is seen in only $20 \%$ to $50 \%$ of the cases [10], in our study $67(74.4 \%)$ of our patients had a history of periumblical or generalized abdominal pain that shifted to the right iliac fossa. This lack of accuracy and improper documentation may be due to the fact that we do not have a clear pathway to follow in managing patients presenting with lower abdominal pain suspected to have acute appendicitis.

Clinical diagnosis and CT scan, in our study, were considered more sensitive than USS, $86 \%, 83 \%$ and $42 \%$, respectively. In addition, the accuracy of both clinical and CT scan were almost close to each other; however, the USS had a very low accuracy result. On the contrary, all of the three tests had almost the same specificity.

\section{Conclusion}

Acute appendicitis shall continue to be a challenging and treacherous disease, especially when it presents in an atypical manner. Its diagnosis is entirely a clinical one provided a good history is taken and a thorough clinical examination is conducted. CT scan and USS as adjunctive diagnostic tools are a real addition to the surgeons' armamentarium, provided they are selectively used based on a clearly written pathway to be strictly followed. The USS is probably restricted to rule out pelvic pathology, help in pregnant ladies, and in children. Our study has limitations which can be overcome by conducting a prospective study to delineate clearly through a well written protocol or pathway to follow the role of good history and thorough clinical examination, and the imaging in the diagnosis of acute appendicitis as well as the atypical presentation of acute appendicitis as lower quadrant abdominal pain.

\section{Acknowledgment}

Dr. Bakr Bin Sadiq Deputy chairman of the research center of KFSHRC Jeddah KSA, for taking the trouble of revising all the statistical data and contributing to its citation in tables and charts. Our librarian Myran for helping out in retrieving most of the literature for this study.

\section{Conflict of interest}

Authors declare no conflict of interest.

\section{References}

1. Nelson DW, Causey MW, Porta CR, McVay DP, Carnes AM, et al. (2013) Examining the relevance of the physician clinical assessment and the reliance on computed tomography in diagnosing acute appendicitis. Am J Surg 205: 452-456. [Crossref]

2. Addiss DG, Shaffer N, Fowler BS, Tauxe RV (1990) The epidemiology of appendicitis and appendectomy in the United States. Am J Epidemiol 132: 910-925. [Crossref]

3. Hale DA, Molloy M, Pearl RH, Schutt DC, Jaques DP (1997) Appendectomy: a contemporary appraisal. Ann Surg 225: 252-261. [Crossref]

4. Lewis FR, Holcroft JW, Boey J, Dunphy E (1975) Appendicitis. A critical review of diagnosis and treatment in ,000 cases. Arch Surg 110: 677-684. [Crossref]

5. Balthazar EJ, Megibow AJ, Siegel SE, Birnbaum BA (1991) Appendicitis: prospective evaluation with high-resolution CT. Radiology 180: 21-24. [Crossref]

6. Birnbaum BA, Jeffrey RB Jr (1998) CT and sonographic evaluation of acute right lower quadrant abdominal pain. AJR Am J Roentgenol 170: 361-371. [Crossref]

7. Jacobs JE (2006) CT and sonography for suspected acute appendicitis: a commentary AJR Am J Roentgenol 186: 1094-1096. [Crossref]

8. Rao PM, Rhea JT, Novelline RA, Mostafavi AA, McCabe CJ (1998) effect of computed tomography of the appendix on treatment of patients and use of hospital resources. $N$ Engl J Med 338: 141-146. [Crossref]

9. Levine CD, Aizenstein O, Lehavi O, Blachar A (2005) Why we miss the diagnosis of appendicitis on abdominal CT: evaluation of imaging features of appendicitis incorrectly diagnosed on CT. AJR Am J Roentgenol 184: 855-859. [Crossref]

10. Lee SL, Walsh AJ, Ho HS (2001) Computed tomography and ultrasonography do not improve and may delay the diagnosis and treatment of acute appendicitis. Arch Surg 136: 556-562. [Crossref]

11. Andersson RE (2004) Meta-analysis of the clinical and laboratory diagnosis of appendicitis. Br J Surg 91: 28-37. [Crossref]

12. Horton MD, Counter SF, Florence MG, Hart MJ (2000) A prospective trial of computed tomography and ultrasonography for diagnosing appendicitis in the atypical patient. Am J Surg 179: 379-381. [Crossref]

13. Franke C, Böhner H, Yang Q, Ohmann C, Röher HD (1999) Ultrasonography for diagnosis of acute appendicitis: results of a prospective multicenter trial. Acute Abdominal Pain Study Group. World J Surg 23: 141-146. [Crossref]

14. Weyant MJ, Eachempati SR, Maluccio MA, Rivadeneira DE, Grobmyer SR, et al (2000) Interpretation of computed tomography does not correlate with laboratory or pathologic findings in surgically confirmed acute appendicitis. Surgery 128: $145-152$. [Crossref]

15. Schwartz SI (1994) Appendix. In: Schwartz SI, ed. Principles of surgery, 6th edn. New York: McGraw Hill 1307-1318.

16. Wilcox RT, Traverso LW (1997) Have the evaluation and treatment of acute appendicitis changed with new technology? Surg Clin North Am 77: 1355-1370. [Crossref]

17. Hong JJ, Cohn SM, Ekeh AP, Newman M, Salama M, et al. (2003) Miami Appendicitis Group. A prospective randomized study of clinical assessment versus computed tomography for the diagnosis of acute appendicitis. Surg Infect (Larchmt) 4: 231-239. [Crossref]

Copyright: (2016 Hamour OA. This is an open-access article distributed under the terms of the Creative Commons Attribution License, which permits unrestricted use, distribution, and reproduction in any medium, provided the original author and source are credited. 